OPEN ACCESS

Edited by:

Dirk Tischler,

Ruhr-Universität Bochum, Germany

Reviewed by:

Peter Neubauer.

Technische Universität Berlin,

Germany

Juan Carlos Aon,

GlaxoSmithKline, United States

*Correspondence:

Gerd M. Seibold

gerd.seibold@uni-ulm.de

Specialty section

This article was submitted to

Microbial Physiology and Metabolism,

a section of the journa

Frontiers in Microbiology

Received: 03 July 2018 Accepted: 08 October 2018

Published: 24 October 2018

Citation:

Goldbeck O, Eck AW and Seibold GM (2018) Real Time Monitoring of NADPH Concentrations in Corynebacterium glutamicum and Escherichia coli via the Genetically Encoded Sensor mBFP. Front. Microbiol. 9:2564. doi: 10.3389/fmicb.2018.02564

\section{Real Time Monitoring of NADPH Concentrations in Corynebacterium glutamicum and Escherichia coli via the Genetically Encoded Sensor mBFP}

\author{
Oliver Goldbeck', Alexander W. Eck ${ }^{2}$ and Gerd M. Seibold ${ }^{1,2 *}$ \\ ${ }^{1}$ Institute of Microbiology and Biotechnology, Ulm University, Ulm, Germany, ${ }^{2}$ Institute for Biochemistry, University \\ of Cologne, Cologne, Germany
}

Analyses of intracellular NADPH concentrations are prerequisites for the design of microbial production strains and process optimization. mBFP was described as metagenomics derived, blue fluorescent protein showing NADPH-dependent fluorescence. Characterization of mBFP showed a high specificity for binding of NADPH $\left(K_{D} 0.64 \mathrm{mM}\right)$ and no binding of $\mathrm{NADH}$, the protein exclusively amplified fluorescence of NADPH. mBFP catalyzed the NADPH-dependent reduction of benzaldehyde and further aldehydes, which fits to its classification as short chain dehydrogenase. For in vivo NADPH analyses a codon-optimized gene for mBFP was introduced into Corynebacterium glutamicum WT and the phosphoglucoisomerase-deficient strain C. glutamicum $\Delta$ pgi, which accumulates high levels of NADPH. For determination of intracellular NADPH concentrations by mBFP a calibration method with permeabilized cells was developed. By this means an increase of intracellular NADPH concentrations within seconds after the addition of glucose to nutrient-starved cells of both C. glutamicum WT and C. glutamicum $\Delta$ pgi was observed; as expected the internal NADPH concentration was significantly higher for C. glutamicum $\Delta p g i(0.31 \mathrm{mM})$ when compared to C. glutamicum WT (0.19 mM). Addition of paraquat to E. coli cells carrying mBFP led as expected to an immediate decrease of intracellular NADPH concentrations, showing the versatile use of mBFP as intracellular sensor.

Keywords: Corynebacterium glutamicum, Escherichia coli, NADPH, redox state, biosensor, short chain dehydrogenase

\section{INTRODUCTION}

The redox state of cells is represented by the ratio of the internal concentrations of the pyridine nucleotides NADH and NADPH and their corresponding oxidized forms $\mathrm{NAD}^{+}$and $\mathrm{NADP}^{+}$ (Blacker et al., 2014). These ubiquitous cofactors are present in cells only in catalytic amounts, therefore efficient recycling is required for the maintenance of viability and antioxidant protection

Abbreviations: CTAB, cetyl trimethylammonium bromide; IPTG, isopropyl $\beta$-D-1-thiogalactopyranoside; TF, transcription factor. 
(Krömer et al., 2008; Agledal et al., 2010; Mailloux et al., 2011). Intracellular availability of NADPH is of special relevance for microbial production processes like the biosynthesis of medicinal compounds, alcohols, biopolymers, and especially amino acids like L-lysine and L-valine (Weckbecker and Hummel, 2004; Sanchez et al., 2006; Bartek et al., 2010; Chemler et al., 2010; Kocharin et al., 2013; Lindner et al., 2013; Zhao et al., 2017). Escherichia coli and Corynebacterium glutamicum, two workhorses for the production of commodity chemicals (Becker and Wittmann, 2015), maintain their NADPH supply mainly via the pentose phosphate pathway (PPP) and the tricarboxylic acid cycle (Marx et al., 1996; Sauer et al., 2004; Spaans et al., 2015). To increase productivity and yields of microbial production strains current metabolic engineering strategies aim at an improved NADPH availability such as the redirection of the metabolism toward the PPP or introduction of transhydrogenases (Kabus et al., 2007; Lindner et al., 2013; Wang et al., 2017). Analyses of intracellular NADPH concentrations and their changes provide the basis for rational strain design and optimization and serve also for the detailed understanding of physiological roles of NADPH (Blacker et al., 2014; Ng et al., 2015; Liu and Wang, 2017). However, research is limited due to the technically challenging and labor-intensive analysis of internal NADPH concentrations (Lu et al., 2018).

Genetically encoded biosensors provide non-invasive optical readouts for internal concentrations of many different metabolites (Bolbat and Schultz, 2017; Lin et al., 2017). These sensors allow high throughput analyses of intracellular metabolite levels in microorganisms as a novel approach for strain selection and can also be employed to monitor metabolite levels in the course of cultivations (Schallmey et al., 2014; Eggeling et al., 2015; Bolbat and Schultz, 2017). For latter application transcription factor (TF) based sensors and the commonly used auto-fluorescent GFP derivatives come along with the drawback of poor dynamic behaviors (Liu et al., 2015; Liu and Wang, 2017), caused by their oxygen dependency and slow response times. For analysis of intracellular NADPH availability, only few biosensors have been described: The [2Fe-2S]-cluster containing transcriptional regulator SoxR of E. coli, was recently used for the design of a NADPH biosensor. Depending on the redox status of SoxR expression of the fluorescent protein eYFP is activated (Siedler et al., 2014). SoxR remains reduced and inactive as long as NADPH-dependent reductases are not limited in their substrate. Lack of NADPH leads to activation of SoxR and thus expression of eYFP. The SoxR-dependent NADPH biosensor was successfully applied to screen $E$. coli strains harboring gene bank derived variants of an NADPH consuming alcohol dehydrogenase (Siedler et al., 2014). A second NADPH sensor named iNAP recently described by Tao et al. (2017) reduces the probability of false positive readouts due to its ratiometric signal. The synthetic iNAP sensor consists of circularly permutated eYFP fused to the NADH binding domain of Rex from Thermus aquaticus (Zhao et al., 2015), of which the nucleotide binding pocket was subsequently mutated for NADPH-specificity by switching conserved residues (Tao et al., 2017). The iNap sensor offers a fast, non-TF-based response to changes in the NADPH/NADP ${ }^{+}$ ratio in various types of cells (Zhao et al., 2016; Tao et al., 2017).

The protein $\mathrm{mBFP}$ was recently described as NADPHdependent, metagenomics derived, blue fluorescent protein: upon binding of NADPH mBFP amplifies the intrinsic fluorescence of NADPH in an oxygen independent manner (Hwang et al., 2012), and produces more fluorescence when supplied with more NADPH (Ng et al., 2015). Oxygen independent fluorescent proteins such as mBFP, whose fluorescent properties rely on the binding of the metabolite of interest, are generally ideal candidates for in vivo analytics of metabolite concentrations in the course of cultivation (Lin et al., 2017). The NADPH dependent fluorescence of mBFP were exploited to screen a series of $E$. coli strains for improved NADPH regeneration (Ng et al., 2015). Based on its amino acid sequence mBFP is classified as short chain dehydrogenase (SDR), however, no experiments were conducted in this direction so far. The analysis of fast variations of intracellular NADPH concentrations by the use of genetically encoded biosensors was to our knowledge hitherto not reported.

In this study, we characterized NADPH binding, fluorescence properties, and enzymatic activities of purified mBFP. Based on this knowledge of its biochemical parameters, we optimized application of this highly specific NADPH sensor for the in vivo analysis of fast changes in intracellular NADPH concentrations in both C. glutamicum and E. coli. The results of these experiments demonstrate that $\mathrm{mBFP}$ is a versatile tool for the quantitative determination of internal NADPH concentrations and their fast alterations in bacteria.

\section{MATERIALS AND METHODS}

\section{Bacterial Strains, Plasmids, and Culture Conditions}

Bacterial strains and plasmids used in this study are listed in Table 1. Pre-cultures of E. coli and C. glutamicum were carried out in 2xTY medium in baffled Erlenmeyer flasks on a rotary shaker $(130 \mathrm{rpm})$ at 30 and $37^{\circ} \mathrm{C}$, respectively. CgXII was used as minimal medium for C. glutamicum (Eggeling and Bott, 2005) with $10 \mathrm{~g} \mathrm{~L}^{-1}$ glucose as carbon source. Strains carrying plasmids were cultivated in the presence of kanamycin $(50 \mu \mathrm{g} / \mathrm{mL})$ and IPTG $(1 \mathrm{mM})$ for $m B F P$ expression. Growth of $E$. coli and of C. glutamicum was followed by measuring the optical density (OD) at $600 \mathrm{~nm}$ in an Ultrospec 2100 pro spectrophotometer (GE Healthcare Life Sciences GmbH, Freiburg, Germany).

\section{Construction of Plasmid pEKEx2_mBFPopt}

For plasmid construction, transformation and plasmid isolation from E. coli $\mathrm{DH} 5 \alpha$ standard cloning and molecular biology procedures were employed (Sambrook et al., 2001), transformation of C. glutamicum strains by electroporation was conducted as described (Tauch et al., 2002). Recombinant strains were selected using 2xTY-agar plates containing kanamycin 
TABLE 1 | Strains and plasmids used in this study

\begin{tabular}{|c|c|c|}
\hline $\begin{array}{l}\text { Strains and } \\
\text { plasmids }\end{array}$ & $\begin{array}{l}\text { Relevant properties and } \\
\text { applications }\end{array}$ & $\begin{array}{l}\text { Sources and } \\
\text { reference }\end{array}$ \\
\hline \multicolumn{3}{|l|}{ Strains } \\
\hline E. coli $\mathrm{DH} 5 \alpha$ & $\begin{array}{l}\mathrm{F}^{-} \text {thi-1 endA1 hsdR17 }\left(\mathrm{r}^{-} \mathrm{m}^{-}\right) \\
\text {supE44 } \Delta \text { lacU169 } \\
\text { ( } \varphi 80 \text { lacZ } \Delta \mathrm{M} 15) \text { recA1 gyrA96 } \\
\text { relA1 } \mathrm{F}^{-} \lambda^{-} \text {ilvG rfb-50 rph-1 }\end{array}$ & Hanahan, 1983 \\
\hline E. coli BL21 (DE3) & $\begin{array}{l}\mathrm{F}^{-} \text {ompT gal dcm lon } \\
\text { hsdSB(rB-mB-) } \\
{[m a l B+] \mathrm{K}-12(\lambda \mathrm{S})}\end{array}$ & $\begin{array}{l}\text { Studier and Moffatt, } \\
1986\end{array}$ \\
\hline $\begin{array}{l}\text { C. glutamicum } \\
\text { ATCC13032 }\end{array}$ & Wild type & $\begin{array}{l}\text { American Type } \\
\text { Culture Collection }\end{array}$ \\
\hline C. glutamicum $\Delta p g i$ & $\begin{array}{l}\text { In-frame deletion of pgi gene } \\
\text { (cg0973) of C. glutamicum } \\
\text { ATCC13032 }\end{array}$ & Lindner et al., 2013 \\
\hline \multicolumn{3}{|l|}{ Plasmids } \\
\hline pEKEx2 & Ptac, $\mid \mathrm{ac} /{ }^{q}, \mathrm{Km}^{\mathrm{r}}$ & $\begin{array}{l}\text { Eikmanns et al., } \\
1991\end{array}$ \\
\hline pCN_mBFP & $\begin{array}{l}\text { Expression plasmid carrying } \\
\text { mBFP under constitutive } \\
\text { promoter }\end{array}$ & Ng et al., 2015 \\
\hline pEKEx2_mBFPopt & $\begin{array}{l}\text { Expression plasmid carrying the } \\
\text { codon-optimized gene for } \\
\text { mBFP under the control of the } \\
\text { IPTG inducible Ptac promoter }\end{array}$ & This work \\
\hline
\end{tabular}

(50 $\mu \mathrm{g} / \mathrm{mL}$ ). Eurofins MWG (Ebersberg, Germany) carried out synthesis of the mBFPopt gene fragment for a C. glutamicum codon-optimized mBFP gene (Supplementary Table S1). The optimized gene was amplified via PCR using the primers mBFPopt_fw and mBFPopt_rev (Supplementary Table S1) resulting in a $780 \mathrm{bp}$ amplicon, which was subsequently digested with the restriction endonucleases SacI and SalI. Ligation of the 766 bp fragment into SacI and SalI linearized pEKEx2 $(8,130 \mathrm{bp})$ resulted in the final construct pEKEx2_mBFPopt $(8,896 \mathrm{bp})$. The plasmid pEKEx2_mBFPopt was controlled by restriction digestions and DNA sequencing (MWG Eurofins).

\section{Fluorescence Analysis}

Fluorescence measurements were carried out in black 96-well plates (Sarstedt, Nümbrecht, Germany) in a TECAN infinite M200 plate reader (Tecan Group, Männedorf, Switzerland) equipped with an injection module. NADPH, NADH and mBFP holoenzyme fluorescence was recorded at an emission of $451 \mathrm{~nm}$ and excitation at $390 \mathrm{~nm}$. For fluorescence kinetic measurements cells were harvested after overnight cultivation by centrifugation (4,000 rpm, $\left.8 \mathrm{~min}, 4^{\circ} \mathrm{C}\right)$, washed twice with $\mathrm{PBS}(137 \mathrm{mM} \mathrm{NaCl}$, $10 \mathrm{mM} \mathrm{Na}_{2} \mathrm{HPO}_{4}, 1.8 \mathrm{mM} \mathrm{NaH} \mathrm{PO}_{4}, \mathrm{pH}$ 7.4) and suspended in $\mathrm{PBS}$ to an $\mathrm{OD}_{600}$ of 1 . Fluorescence kinetic measurements were performed using read intervals of 1-2 $s$ and automatic injection of substrate ( $100 \mathrm{mM}$ glucose final concentration) at indicated time points. For kinetic measurements of the effects of paraquat first glucose (final concentration $100 \mathrm{mM}$ ) was added after $30 \mathrm{~s}$ of pre-incubation to cells suspended in PBS and then at the indicated time point paraquat $(8-16 \mathrm{mM}$ final concentration) was added. For analysis of mBFP fluorescence during cultivation of C. glutamicum, cultures were sampled after $2 \mathrm{~h}$ of cultivation, and the $\mathrm{OD}_{600}$ of the sample set to 1 with CgXII minimal medium before the fluorescence was measured in the plate reader.

Fluorescence microscopy was performed with an Axio Observer Z1 microscope (Zeiss, Oberkochen, Germany) using Zen software. For the visualization of mBFP fluorescence, cells were cultivated and treated as described above for kinetic measurements; $2 \%(\mathrm{w} / \mathrm{v})$ glucose (final concentration) were added immediately before immobilization on agarose pads $(1 \% \mathrm{w} / \mathrm{v}$ of agarose in PBS). For detection of mBFP fluorescence the Zeiss filter set 49 (Excitation 365 nm, Emission $445 / 50 \mathrm{~nm}$ ) was used. To analyze permeabilization of E. coli and C. glutamicum cell membranes by CTAB treatment, the LIVE/DEAD ${ }^{\mathrm{TM}}$ BacLight $^{\mathrm{TM}}$ Bacterial Viability Kit was used according to the manufacturer's instructions (Thermo Scientific, Waltham, MA, United States) and the AF microscope filter to analyze propidium iodide staining of the permeabilized cell (excitation at $545 \pm 30 \mathrm{~nm}$, emission at $610 \pm 75 \mathrm{~nm}$ ). Cells were cultivated and washed as described above for kinetic fluorescence measurements, CTAB was added to the washed cells at concentrations indicated in results $5 \mathrm{~min}$ prior before samples were analyzed by microscopy.

For in situ calibration of the sensor signals, cells were harvested and washed with PBS as described above for kinetic fluorescence measurements. The washed cells were transferred to black 96-well plates and $0.05 \%(\mathrm{w} / \mathrm{v})$ CTAB (final concentration) were added. After $2 \mathrm{~min}$ of incubation at room temperature, NADPH at concentrations from 0.01 to $1 \mathrm{mM}$ was added to the CTAB treated cells and subsequently fluorescence was measured at 395 and $451 \mathrm{~nm}$ for excitation and emission, respectively. Thereby obtained fluorescence values were plotted against the NADPH concentrations and the resulting calibration curve was then used for the quantification of the intracellular NADPH concentrations in the fluorescence kinetic measurements described above.

\section{In vitro Characterization of mBFP}

For purification of mBFP E. coli ( $\left.\mathrm{pCN} \_m B F P\right)$ cells were cultivated in TB-medium (Hobbs and Tartoff, 1987) to an $\mathrm{OD}_{600}$ of 12. Cells were harvested by centrifugation $(20 \mathrm{~min}, 3,200 \times g$, $4^{\circ} \mathrm{C}$, Eppendorf $5804 \mathrm{R}$ Centrifuge), washed twice with start buffer (50 mM KH${ }_{2} \mathrm{PO}_{4}, \mathrm{pH} 7$ ), suspended in start buffer, and disrupted using a Branson Sonifier 250 (Branson Ultrasonics, Danbury, CT, United States). Amplitude was set to $90 \%$, cycle 0.5 and 10 times $30 \mathrm{~s}$ burst intervals with intermittent cooling on ice were performed. After removal of cell debris by centrifugation $\left(16,000 \times g, 4^{\circ} \mathrm{C}, 15 \mathrm{~min}\right.$, Eppendorf $5804 \mathrm{R}$ Centrifuge $)$ the supernatant was centrifuged at $60,000 \times g, 4^{\circ} \mathrm{C}, 1 \mathrm{~h}($ Beckmann XPN 100 ultracentrifuge) to remove the membrane fraction. The cytosolic fraction was diluted 1:3 with start buffer and applied to a HiScreen Capto Blue column (GE Healthcare) equilibrated with start buffer on an Äkta Purifier chromatography system (GE Healthcare). After washing the column with $40 \mathrm{~mL}$ start buffer to remove unspecific bound proteins, bound proteins were eluted using a gradient step with elution buffer $(1.5 \mathrm{M} \mathrm{KCl}, 50 \mathrm{mM}$ 
$\mathrm{KH}_{2} \mathrm{PO}_{4}, \mathrm{pH}$ 7). Fractions were collected, screened by activity and fluorescence analysis, and analyzed by SDS-PAGE according to Laemmli (1970).

Thermoshift assays were done in a CFX96 ${ }^{\mathrm{TM}}$ real-time PCR detection system (BioRad Laboratories, Hercules, CA, United States) using SYPRO orange fluorescent dye (Sigma Aldrich, St. Louis, MO, United States) as described (Niesen et al., 2007).

Fluorescence of the purified $\mathrm{mBFP}$ in absence and presence of NADPH was determined as described above in an TECAN infinite M200 plate reader at an emission of $451 \mathrm{~nm}$ and excitation at $390 \mathrm{~nm}$.

Enzymatic activity of mBFP with different substrates and cofactors was determined using an Ultrospec 2100pro photometer (GE Healthcare). The reaction was performed at $30^{\circ} \mathrm{C}$ in phosphate buffer $\left(0.4 \mathrm{~g} / \mathrm{L} \mathrm{KH}_{2} \mathrm{PO}_{4}, 12.6 \mathrm{~g} / \mathrm{L} \mathrm{K}_{2} \mathrm{HPO}_{4}, \mathrm{pH}\right.$ 8). NADPH and NADH were used in concentrations of $200 \mu \mathrm{M}$ for the determination of benzaldehyde dependent activity (or other substrates, see Supplementary Material). Varying benzaldehyde concentrations were used for the determination of kinetic parameters $\left(K_{\mathrm{M}}, k_{\text {cat }}\right)$. Protein concentrations were determined with the Roti-Nanoquant kit (Carl Roth, Karlsruhe, Germany) using a BSA standard.

\section{RESULTS AND DISCUSSION}

\section{The NADPH Sensor Protein mBFP Possesses Benzaldehyde Reductase Activity}

Genetically encoded biosensors offer various opportunities for analysis of intracellular metabolites in microorganisms in the course of cultivation and the development of novel strain selection strategies (Eggeling et al., 2015; Cheng et al., 2018). Fluorescent proteins such as mBFP, whose fluorescent properties exclusively rely on the binding of the metabolite of interest, are good candidates for in vivo analytics. The metagenome derived blue fluorescent protein $\mathrm{mBFP}$ exhibits blue fluorescence upon binding of NADPH independently of the availability of oxygen (Hwang et al., 2012). mBFP was here produced with E. coli (pCN_mBFP), which carries the plasmid pCN_mBFP for constitutive high expression of $m B F P$ in E. coli strains (Spaans et al., 2015). After cell growth, harvest and disruption the mBFP protein was purified from cell free extracts of E. coli (pCN_mBFP) to apparent homogeneity by affinity chromatography using a HiScreen Capto Blue column (Supplementary Figure S1). Previous studies on $\mathrm{mBFP}$ disregarded the possibility that mBFP may possess an enzymatic function (Polizzi et al., 2007; Buysschaert et al., 2013) albeit it shares high sequence similarities with other, well characterized, enzymatically active SDR (Supplementary Figure S2). We tested purified mBFP with different known substrates of SDRs thereby using either NADPH or NADH as coenzyme. Benzaldehyde in combination with NADPH as cofactor was found to be the preferred substrate of mBFP (Supplementary Table S2). Minor enzyme activities were also detected for $\mathrm{mBFP}$ with a series of different other aldehydes as substrates (Supplementary Table S2), however, activities of mBFP with different substrates were detected exclusively with $\mathrm{NADPH}$ as a cofactor. Further analysis of $\mathrm{mBFP}$ activity with different amounts of the substrate benzaldehyde and the cofactor NADPH revealed saturation kinetics for both compounds. The activity data were fitted to Michaelis-Menten kinetics and resulted in a $K_{\mathrm{M}}$ value of $3.1 \mathrm{mM}$ of $\mathrm{mBFP}$ for benzaldehyde (Figure 1A) and a $k_{\text {cat }}$ of $4.51 / \mathrm{s}$. Analysis of the mBFP activity data with different NADPH concentrations resulted in a $K_{\mathrm{M}}$ value of $41 \mu \mathrm{M}$ for NADPH and a $k_{\text {cat }}$ of $11.851 / \mathrm{s}$ (Figure 1B). With $\mathrm{NADH}$ as co-factor, no enzymatic activity of mBFP was detected. From these results, we conclude that $\mathrm{mBFP}$ is an active reductase with a preference for NADPH as cofactor.

\section{mBFP Exclusively Binds NADPH}

The quality of a metabolite sensor stands or falls with the specificity of the signal induced by the detected molecule. Noticeable, mBFP amplifies the intrinsic fluorescence of NADPH upon binding as it is described for other SDRs (Polizzi et al., 2007; Buysschaert et al., 2012). As expected purified mBFP apoenzyme shows no emission at $451 \mathrm{~nm}$ when excited at $395 \mathrm{~nm}$, but its presence lead to an increase of the emission signal for $0.5 \mathrm{mM}$ $\mathrm{NADPH}$ about 8.5 fold from $4,532 \pm 408$ (pure NADPH) to $38,402 \pm 277$ (NADPH $+\mathrm{mBFP}$ ) (Figure 2). For NADH no amplification of its intrinsic fluorescence by addition of mBFP was detected (Figure 2), demonstrating the specificity of the mBFP for NADPH.

To analyze mBFPs affinity for different cofactors, thermal degradation assays (thermofluor) were performed. In these experiments binding of a cofactor leads to a shift in melting temperature $\left(T_{\text {Melt }}\right)$ of the protein under investigation (Niesen et al., 2007). The $T_{\text {Melt }}$ of $47.5^{\circ} \mathrm{C}$ determined for the mBFP apoenzyme was shifted to $55^{\circ} \mathrm{C}$ upon addition of $1 \mathrm{mM} \mathrm{NADPH}$ and $52^{\circ} \mathrm{C}$ upon addition of $1 \mathrm{mM} \mathrm{NADP}^{+}$(Figure $3 \mathrm{~A}$ and Supplementary Figure S3A), which implies a higher affinity of $\mathrm{mBFP}$ toward NADPH than $\mathrm{NADP}^{+}$. Binding of mBFP to $\mathrm{NADPH}$ and NADP is plausible as redox enzymes often catalyze reversible reactions. For further characterization, effects of different NADPH concentrations $(0-10 \mathrm{mM})$ on $T_{\text {Melt }}$ of mBFP were analyzed (Figure 3B). Non-linear regression of the resulting data revealed a saturation kinetics with a dissociation constant $\left(K_{\mathrm{D}}\right)$ of $0.64 \mathrm{mM}$ (Figure 3B and Supplementary Figure S3B). Interestingly only presence of phosphorylated cofactors led to an increase in $T_{\text {Melt }}$ of $\mathrm{mBFP}$, whereas $T_{\text {Melt }}$ determined in presence of the non-phosphorylated cofactors NADH $\left(47^{\circ} \mathrm{C}\right)$ and $\mathrm{NAD}^{+}$ $\left(47.5^{\circ} \mathrm{C}\right)$ were identical to $T_{\text {Melt }}$ of the apoenzyme (Figure $3 \mathrm{~A}$ ). This result indicates that $\mathrm{mBFP}$ does not bind to NADH and $\mathrm{NAD}^{+}$. The broad dynamic range of fluorescence amplification in combination with the high specificity for NADPH makes mBFP a suitable candidate to be used as NADPH biosensor for in vivo applications.

\section{Use of the Genetically Encoded NADPH Sensor mBFP in C. glutamicum}

Large-scale microbial production of amino acids such as L-lysine heavily relies on efficient recycling of the cofactor 

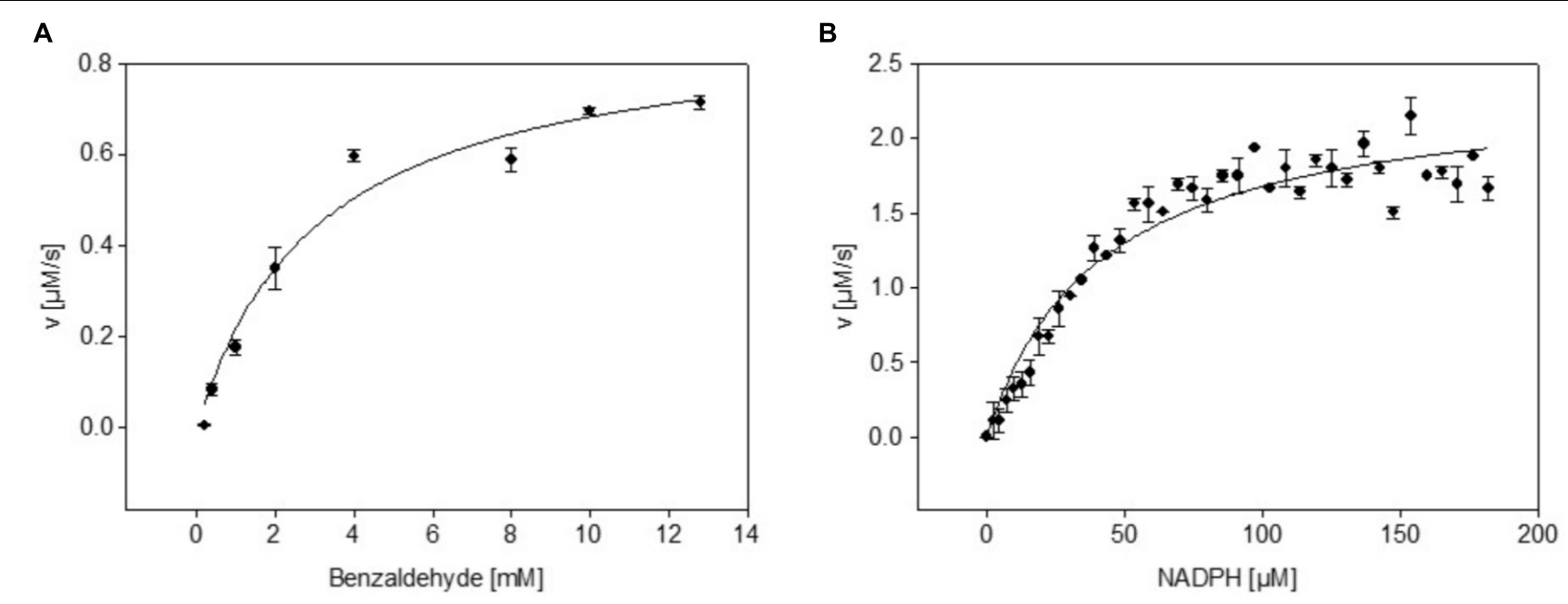

FIGURE 1 | Dependence of dehydrogenase activity of purified mBFP from its substrate benzaldehyde (A) and its cofactor NADPH (B); different concentrations of benzaldehyde (0.5-12.5 mM; (A) and NADPH (0.5-180 $\mu \mathrm{M}$; (B) were tested in presence of $200 \mu \mathrm{M}$ NADPH and 12.5 mM benzaldehyde, respectively. Data represent mean values from three independent measurements, data were fitted according to the Michaelis-Menten equation.

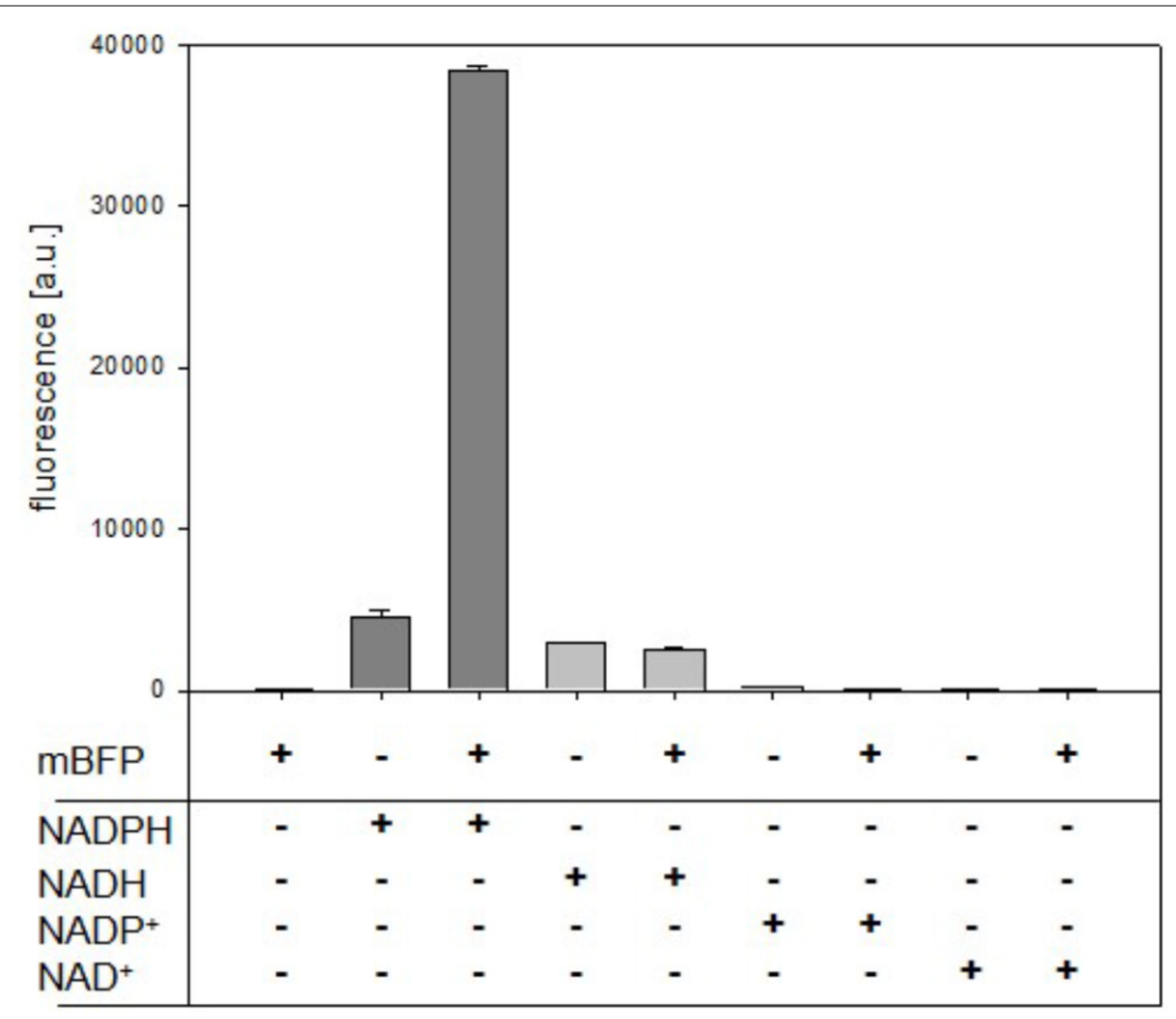

FIGURE 2 | Effects of purified mBFP on fluorescence of NADPH, NADH, NADP ${ }^{+}$, and NAD+ (each at $0.5 \mathrm{mM}$ ) at excitation with $395 \mathrm{~nm}$ and emission at $451 \mathrm{~nm}$. Data represent mean values and SDs of three independent measurements. 

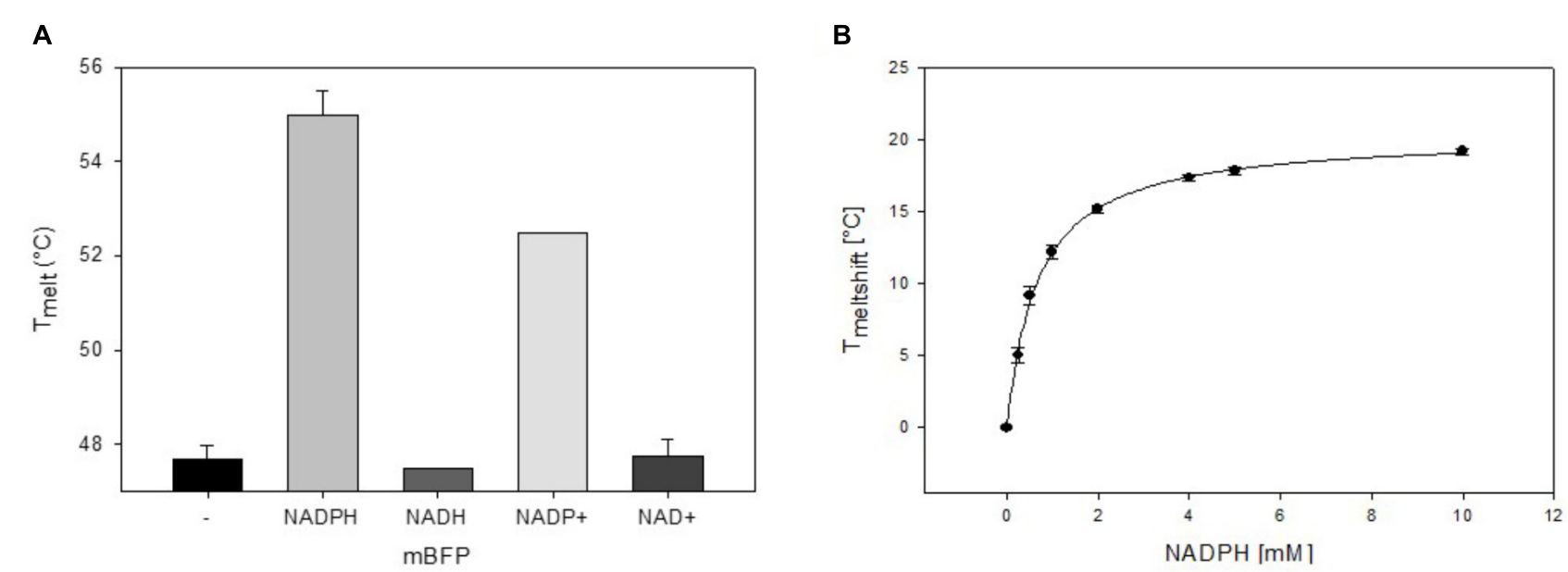

FIGURE 3 | Thermal shift assays for the analysis of the cofactor preference of mBFP. Melting temperatures of apo-mBFP without (-) or in the presence of (1 mM each) $\mathrm{NADPH}, \mathrm{NADH}, \mathrm{NADP}^{+}$, or $\mathrm{NAD}^{+} \mathbf{( A )}$. Dependence of mBFP melting temperature on the concentration of its cofactor NADPH (B), NADPH concentrations of 0-10 mM were tested. Melting temperatures were determined by heating from 40 to $70^{\circ} \mathrm{C}$ in $0.5^{\circ} \mathrm{C}$ steps, and unfolding was monitored as described in Section "Materials and Methods." Data represent mean values and SDs of three independent measurements, data in (B) were fitted according to the Michaelis-Menten equation.

NADPH (Eggeling and Bott, 2015; Xu et al., 2018), which has therefore been a well-studied target for the engineering of C. glutamicum strains (Blombach and Seibold, 2010; Wang et al., 2016). For analysis of NADPH levels in C. glutamicum via $\mathrm{mBFP}$ as a genetically encoded sensor, the plasmid pEKEx2_mBFPopt was constructed. For this purpose, an mBFP gene was synthesized with codon-optimization for C. glutamicum and cloned into the plasmid pEKEx2. The sensor plasmid was introduced in C. glutamicum WT and the phosphoglucoisomerase-deficient mutant strain C. glutamicum $\Delta p g i$. Deletion of $p g i$ results in the redirection of the carbon flux from glycolysis to the pentose-phosphate pathway, which brings about the generation of high NADPH concentrations in this strain (Marx et al., 2003). To analyze NADPH concentrations with the sensor mBFP, cells of C. glutamicum WT (pEKEx2_mBFPopt) and C. glutamicum $\triangle p g i$ (pEKEx2_mBFPopt) were cultivated in CgXII medium with $100 \mathrm{mM}$ glucose as substrate, sampled after $4 \mathrm{~h}$ of cultivation, and their fluorescence analyzed in a plate reader. A significantly higher fluorescence of $2,125 \pm 551$ FLU/OD at excitation $395 \mathrm{~nm}$ with and emission at $451 \mathrm{~nm}$ was detected for C. glutamicum $\triangle p g i$ (pEKEx2_mBFPopt) when compared to $C$. glutamicum (pEKEx2_mBFPopt), for which a fluorescence of $613 \pm 167$ FLU/OD was determined.

Analyses of these cells by fluorescence microscopy revealed besides the overall increased fluorescence of C. glutamicum $\triangle p g i$ (pEKEx2_mBFPopt) when compared to C. glutamicum (pEKEx2_mBFPopt) that the fluorescence showed only minor cell-cell variations for each strain (Supplementary Figure S4). As for C. glutamicum $\triangle p g i$ an increased NADPH concentration is expected during cultivation with glucose when compared to C. glutamicum WT, the observed higher overall fluorescence for C. glutamicum $\triangle p g i$ (pEKEx2_mBFPopt) indicates that by the use of mBFP NADPH levels can be monitored in C. glutamicum.

\section{mBFP Enables Detection of Fast Alterations of NADPH Concentrations in C. glutamicum and E. coli}

Current techniques for analyses of intracellular NADPH concentrations are mostly employed to study steady state levels but have a limited capacity for analyses of dynamic changes. However, the latter are the key for the understanding of sensing and adaptive mechanisms, drug modes of action, and homeostasis mechanisms (Zampieri et al., 2017). In bacteria, levels of metabolites often change upon perturbations within seconds (Chubukov et al., 2014; Wegner et al., 2015). These fast changes cannot be observed in real-time by the use of genetically encoded sensors requiring transcription and translation of a fluorescent reporter, but the properties of protein based sensors are matching this task (Liu et al., 2015; Lin et al., 2017). The protein sensor $\mathrm{mBFP}$ binds and enhances specifically the fluorescence of NADPH and was above shown to be suited for measurements of intracellular NADPH concentrations in C. glutamicum.

To test utilization of mBFP for the detection of fast changes of NADPH levels, the response to fast changes of nutrient availability was analyzed in C. glutamicum strains carrying mBFP. C. glutamicum WT (pEKEx2_mBFPopt) and C. glutamicum $\triangle p g i$ (pEKEx2_mBFPopt) were cultivated for $12 \mathrm{~h}$ in $2 x T Y$ complex medium with $1 \mathrm{mM}$ IPTG to induce synthesis of mBFP. The derived nutrient-starved cells of C. glutamicum WT (pEKEx2_mBFPopt) and C. glutamicum $\Delta p g i$ (pEKEx2_mBFPopt) were washed twice with PBS, suspended in PBS and transferred into single wells of a 96-well plate. 
Kinetic assays were performed in a Tecan M200 plate reader equipped with an injector module: mBFP fluorescence was read in $2 \mathrm{~s}$ intervals for $2 \mathrm{~min}$ and glucose was injected automatically into the wells after the first $15 \mathrm{~s}$ of the kinetic assay. As depicted in Figure $\mathbf{4 A}$, a very fast increase of the mBFP fluorescence within $30-40 \mathrm{~s}$ after the addition of glucose to 658 FLU and 1,374 FLU can be seen for C. glutamicum WT (pEKEx2_mBFPopt) and C. glutamicum $\triangle p g i$ (pEKEx2_mBFPopt), respectively. As the addition of glucose and its metabolization via glycolysis and tricarboxylic acid cycle besides NADPH probably also causes formation of the redox intermediates $\mathrm{NADH}$ and FADH (Blombach and Seibold, 2010), which both also show fluorescence at an emission wavelength of $451 \mathrm{~nm}$ when excited at $390 \mathrm{~nm}$, changes of fluorescence upon glucose addition were also analyzed for cells of the control strains C. glutamicum WT (pEKEx2) and C. glutamicum $\Delta p g i$ (pEKEx2). As depicted in Supplementary Figure S5 the fluorescence increased after the addition of glucose from initially 14 FLU to 19 FLU and from 10 FLU to 50 FLU in C. glutamicum WT (pEKEx2) and C. glutamicum $\triangle p g i$ (pEKEx2), respectively. As fluorescence values obtained for the two mBFP carrying strains C. glutamicum WT (pEKEx2_mBFPopt) and C. glutamicum $\triangle p g i$ (pEKEx2_mBFPopt) are about 30 times higher than the fluorescence values measured for the mBFP-deficient control strain, the amplification of $\mathrm{NADPH}$ fluorescence by $\mathrm{mBFP}$ effectively masks the fluorescence derived from other redox intermediates such as $\mathrm{NADH}$ and $\mathrm{FADH}$ in the mBFP carrying strains.

For determination of intracellular NADPH concentrations we calibrated the sensor in situ in cells of C. glutamicum WT (pEKEx2_mBFPopt) and C. glutamicum $\triangle p g i$ (pEKEx2_mBFPopt), which were permeabilized by addition of $0.05 \%(\mathrm{w} / \mathrm{v})$ of CTAB (final concentration). At this CTAB concentration small pores are formed in the membrane, which allow the fast diffusion of small molecules, however, the cell's superstructure remains intact (Supplementary Figure S6). As depicted in Figure 4B, different slopes were observed for the linear regression lines based $\mathrm{NADPH}$ dependent mBFP fluorescence of permeabilized cells of C. glutamicum WT (pEKEx2_mBFPopt) and C. glutamicum $\triangle p g i$ (pEKEx2_mBFPopt), which is probably brought about different levels of $\mathrm{mBFP}$ formed in course of the pre-cultivation. Based on the in situ calibration for C. glutamicum WT (pEKEx2_mBFPopt) an increase of the NADPH concentration from initially 0.127 to $0.185 \mathrm{mM}$ after the addition of glucose was determined (Figure 4C). The initial NADPH concentration of $0.175 \mathrm{mM}$ determined for starved cells of C. glutamicum $\triangle p g i$ (pEKEx2_mBFPopt) is higher than the initial concentration determined for C. glutamicum WT (pEKEx2_mBFPopt), and after glucose addition the NADPH concentration in C. glutamicum $\triangle p g i$ (pEKEx2_mBFPopt) increased up to $0.313 \mathrm{mM}$. The intracellular concentrations already reached within $1 \mathrm{~min}$ after the addition of the substrate in both C. glutamicum WT (pEKEx2_mBFPopt) and C. glutamicum $\triangle p g i$ (pEKEx2_mBFPopt) are in the same concentration range as the NADPH concentrations

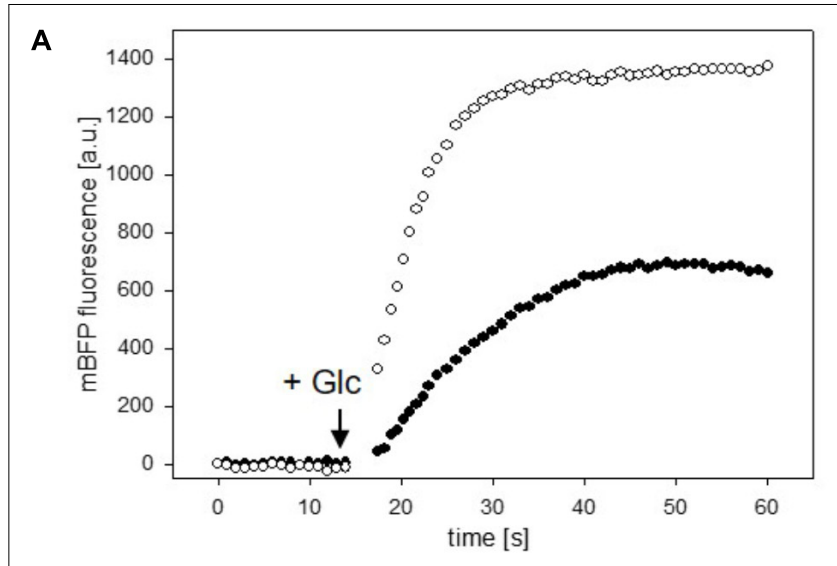

B

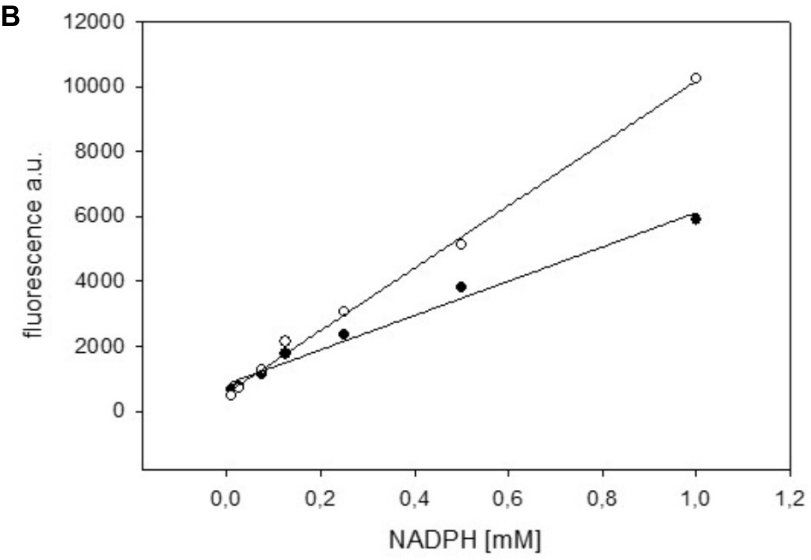

C

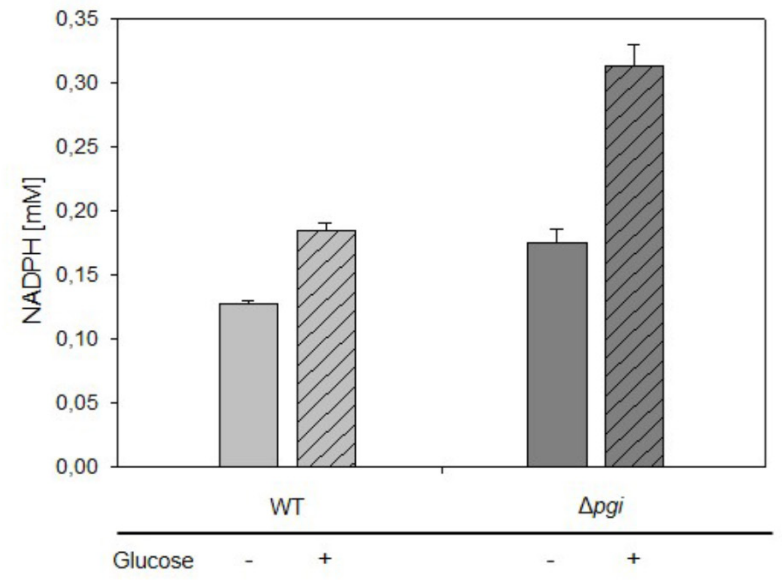

FIGURE 4 | Analyses of changes of mBFP fluorescence in starved cells of C. glutamicum WT (pEKEx2-mBFPopt) [filled circles] and C. glutamicum $\Delta p g i$ (pEKEx2-mBFPopt) [open circles] upon addition of the substrate glucose (indicated by the arrow) (A). In situ calibration of mBFP derived signals by the use CTAB permeabilized cells from C. glutamicum WT (pEKEx2-mBFPopt) [filled circles] and C. glutamicum $\Delta$ pgi (pEKEx2-mBFPopt) [open circles] (B) in presence of different amounts of added NADPH. Steady state levels of intracellular NADPH concentrations calculated based on in situ calibrations in C. glutamicum WT (pEKEx2-mBFPopt) and C. glutamicum $\triangle$ pgi (pEKEx2-mBFPopt) before and after glucose addition (C). For panels (A,B) one representative experiment of a series of three independent experiments is shown. Data in $\mathbf{( C )}$ represent mean values and SDs of three independent experiments. 
determined via metabolite extraction and subsequent HPLC-MS analyses for WT and Pgi-deficient C. glutamicum cells, respectively, in previous studies (Marx et al., 2003; Bartek et al., 2010).

Versatility of the mBFP-based method for observation of fast variations of NADPH concentrations was also tested in E. coli DH5 $\alpha$ (pEKEx2_mBFPopt). For this purpose, cells of E. coli $\mathrm{DH} 5 \alpha$ (pEKEx2_mBFPopt) cultivated in 2 XTY medium and induced with $1 \mathrm{mM}$ IPTG for $12 \mathrm{~h}$ were harvested by centrifugation and carefully washed with PBS. One aliquot of the washed E. coli DH5 $\alpha$ (pEKEx2_mBFPopt) cells was permeabilized by addition of $0.05 \%$ CTAB (Supplementary Figure S7) and then used for an in situ calibration of the $\mathrm{mBFP}$ sensor signal response to different $\mathrm{NADPH}$ concentrations (Figure 5B). Further aliquots of E. coli $\mathrm{DH} 5 \alpha$ (pEKEx2_mBFPopt) were transferred to wells of a 96-well plate and used for kinetic assays. Within $30 \mathrm{~s}$ upon addition of $100 \mathrm{mM}$ glucose (final concentration) the intracellular NADPH concentration increased in E. coli DH5 $\alpha$ (pEKEx2_mBFPopt) from 140 to $180 \mu \mathrm{M}$, which shows that in both $E$. coli and C. glutamicum NADPH accumulates fast in response to increases of nutrient availability. Protein based sensors like mBFP should also enable the observation of fast decreases in their analytes concentrations. Addition of paraquat initiates antioxidative mechanisms in E. coli, which lead to the depletion of the NADPH pool (Gu and Imlay, 2011). Indeed, upon addition of paraquat to glucose-fed cells of E. coli DH5 $\alpha$ (pEKEx2_mBFPopt) the intracellular NADPH concentrations decreased in dependence of the added paraquat concentration (Figure 5A). As expected addition of water did not lead to a change of the intracellular $\mathrm{NADPH}$ concentration in control experiments (Figure 5A). These results demonstrate the functionality of $\mathrm{mBFP}$ in two different hosts and show that it is highly suitable for the fast dynamic measurements of intracellular NADPH concentrations required to understand redox mechanisms of cells.

\section{CONCLUSION}

mBFP was here shown to specifically bind to and amplify fluorescence of NADPH, which are essential prerequisites for its use as a genetically encoded biosensor for the analysis of intracellular NADPH concentrations. Plasmid encoded mBFP allowed to measure fast changes of NADPH concentrations in both C. glutamicum and E. coli strains and most importantly it allowed to analyze increases as well as decreases of NADPH concentrations in real time. It has to be denoted, that each use of $\mathrm{mBFP}$ requires calibration of the signals by permeabilization of the cell and incubation with different NADPH concentrations as the mBFP derived fluorescence signal depends both on the internal NADPH concentration as well as the amount of mBFP, which also varies. For short time intervals variations of mBFP amounts can be neglected but to monitor intracellular NADPH levels in the course of fermentations regular sampling to perform calibrations with permeabilized cells is probably necessary. The development of a ratiometric sensor based on mBFP will probably allow to easily monitor NADPH levels also during longtime experiments. The monitoring of NADPH levels in C. glutamicum and E. coli during cultivations is interesting as NADPH is the redox cofactor limiting production of bulk chemicals synthesized via anabolic pathways such as amino acids (Hirasawa and Shimizu, 2016; Xu et al., 2018), but for this purpose conventional, sampling dependent techniques can be easily employed (Liu et al., 2018). The general advantage of a genetically encoded metabolite sensors for NADPH like $\mathrm{mBFP}$ is the site specific analyses of metabolite concentrations, e.g., in to monitor compartment-specific transient changes of

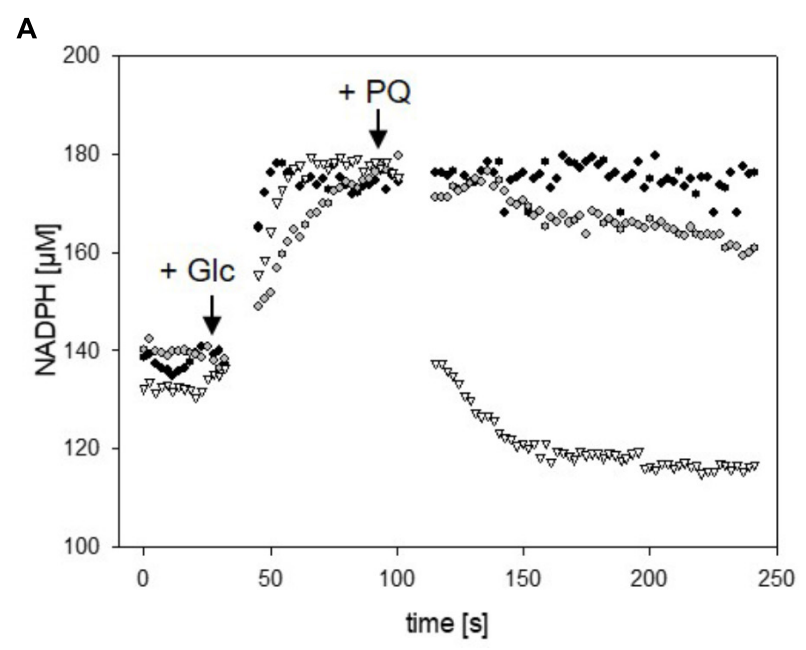

B

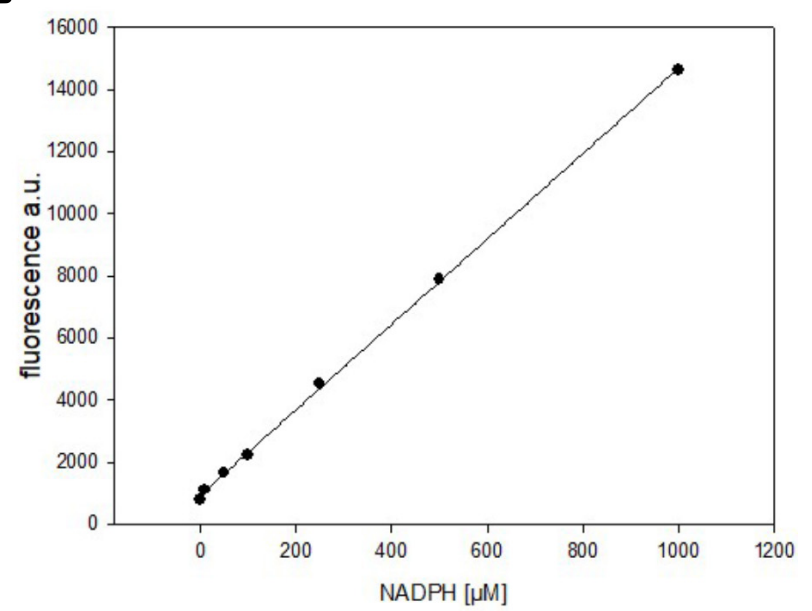

FIGURE 5 | Analyses of changes of NADPH concentrations in cells of E. coli DH5 $\alpha$ (pEKEx2_mBFPopt) upon addition of the substrate glucose (indicated by the arrow, $100 \mathrm{mM}$ ) and consecutive addition of different amounts of paraquat ( $8 \mathrm{mM}$ paraquat - gray circles, $16 \mathrm{mM}$ paraquat - white triangles, no addition of paraquat-black circles) (A). In situ calibration of mBFP derived signals by the use CTAB permeabilized cells from E. coli DH (pEKEx2_mBFPopt) in presence of different amounts of added NADPH (B). One representative experiment of a series of three independent experiments is shown. 
intracellular balances of NADPH in eukaryotic cells (Ying, 2008; Wiederkehr and Demaurex, 2017). These measurements are currently performed via analyses of autofluorescence (Blacker et al., 2012; Blacker and Duchen, 2016), which unfortunately coincidences in the case of NADH and NADPH. The specificity of $\mathrm{mBFP}$ for $\mathrm{NADPH}$ over $\mathrm{NADH}$, the masking of $\mathrm{NADH}$ fluorescence by the strong amplification of the NADPH fluorescence signal, and the possibility to monitor fast changes without sampling indicate that mBFP might be a suitable molecular tool for cell biologist. In its present state, the sensor protein $\mathrm{mBFP}$ is already a well suited to observe fast changes of intracellular NADPH concentrations in bacteria, which provides a new insight into the kinetics of microbial metabolism.

\section{AUTHOR CONTRIBUTIONS}

GS and OG designed and coordinated the study. OG and AE carried out the experiments. GS, AE, and OG analyzed the results and wrote the manuscript.

\section{REFERENCES}

Agledal, L., Niere, M., and Ziegler, M. (2010). The phosphate makes a difference: cellular functions of NADP. Redox Rep. 15, 2-10. doi: 10.1179/ 174329210X12650506623122

Bartek, T., Blombach, B., Zonnchen, E., Makus, P., Lang, S., Eikmanns, B. J., et al. (2010). Importance of NADPH supply for improved L-valine formation in Corynebacterium glutamicum. Biotechnol. Prog. 26, 361-371. doi: 10.1002/btpr. 345

Becker, J., and Wittmann, C. (2015). Advanced biotechnology: metabolically engineered cells for the bio-based production of chemicals and fuels, materials, and health-care products. Angew. Chem. Int. Ed. Engl. 54, 3328-3350. doi: 10.1002/anie. 201409033

Blacker, T. S., and Duchen, M. R. (2016). Investigating mitochondrial redox state using NADH and NADPH autofluorescence. Free Radic. Biol. Med. 100, 53-65. doi: 10.1016/j.freeradbiomed.2016.08.010

Blacker, T. S., Mann, Z. F., Gale, J. E., Ziegler, M., Bain, A. J., and Duchen, M. R. (2012). Separation of NADPH and NADH fluorescence emission in live cells using flim. Biophys. J. 102, 196a. doi: 10.1016/j.bpj.2011. 11.1067

Blacker, T. S., Mann, Z. F., Gale, J. E., Ziegler, M., Bain, A. J., Szabadkai, G., et al. (2014). Separating NADH and NADPH fluorescence in live cells and tissues using FLIM. Nat. Commun. 5:3936. doi: 10.1038/ncomms4936

Blombach, B., and Seibold, G. M. (2010). Carbohydrate metabolism in Corynebacterium glutamicum and applications for the metabolic engineering of L-lysine production strains. Appl. Microbiol. Biotechnol. 86, 1313-1322. doi: 10.1007/s00253-010-2537-z

Bolbat, A., and Schultz, C. (2017). Recent developments of genetically encoded optical sensors for cell biology. Biol. Cell 109, 1-23. doi: 10.1111/boc.2016 00040

Buysschaert, G., Verstraete, K., Savvides, S. N., and Vergauwen, B. (2012). Crystallization of an atypical short-chain dehydrogenase from Vibrio vulnificus lacking the conserved catalytic tetrad. Acta Crystallogr. Sect. F Struct. Biol. Cryst. Commun. 68, 771-774. doi: 10.1107/S1744309112018672

Buysschaert, G., Verstraete, K., Savvides, S. N., and Vergauwen, B. (2013). Structural and biochemical characterization of an atypical short-chain dehydrogenase/reductase reveals an unusual cofactor preference. FEBS J. 280, 1358-1370. doi: 10.1111/febs.12128

Chemler, J. A., Fowler, Z. L., Mchugh, K. P., and Koffas, M. A. (2010). Improving NADPH availability for natural product biosynthesis in Escherichia coli by metabolic engineering. Metab. Eng. 12, 96-104. doi: 10.1016/j.ymben.2009.07. 003

\section{FUNDING}

We thank the German Ministry of Education and Research for financial funding in the frame of the e:Bio initiative (Contract No. 031A302D).

\section{ACKNOWLEDGMENTS}

We thank Bernhard Eikmanns and Reinhard Kärmer for continuous support. We are very grateful to Howard Salis for the generous gift of the plasmid pCN-mBFP (Addgene plasmid \#63847).

\section{SUPPLEMENTARY MATERIAL}

The Supplementary Material for this article can be found online at: https://www.frontiersin.org/articles/10.3389/fmicb. 2018.02564/full\#supplementary-material

Cheng, F., Tang, X. L., and Kardashliev, T. (2018). Transcription factorbased biosensors in high-throughput screening: advances and applications. Biotechnol. J. 13:e1700648. doi: 10.1002/biot.201700648

Chubukov, V., Gerosa, L., Kochanowski, K., and Sauer, U. (2014). Coordination of microbial metabolism. Nat. Rev. Microbiol. 12, 327-340. doi: 10.1038/ nrmicro3238

Eggeling, L., and Bott, M. (2005). Handbook of Corynebacterium glutamicum. Boca Raton, FL: CRC Press. doi: 10.1201/9781420039696

Eggeling, L., and Bott, M. (2015). A giant market and a powerful metabolism: L-lysine provided by Corynebacterium glutamicum. Appl. Microbiol. Biotechnol. 99, 3387-3394. doi: 10.1007/s00253-015-6508-2

Eggeling, L., Bott, M., and Marienhagen, J. (2015). Novel screening methodsbiosensors. Curr. Opin. Biotechnol. 35, 30-36. doi: 10.1016/j.copbio.2014.12. 021

Eikmanns, B. J., Kleinertz, E., Liebl, W., and Sahm, H. (1991). A family of Corynebacterium glutamicum/Escherichia coli shuttle vectors for cloning, controlled gene expression, and promoter probing. Gene 102, 93-98. doi: 10. 1016/0378-1119(91)90545-M

Gu, M., and Imlay, J. A. (2011). The SoxRS response of Escherichia coli is directly activated by redox-cycling drugs rather than by superoxide. Mol. Microbiol. 79, 1136-1150. doi: 10.1111/j.1365-2958.2010.07520.x

Hanahan, D. (1983). Studies on transformation of Escherichia coli with plasmids. J. Mol. Biol. 166, 557-580. doi: 10.1016/S0022-2836(83)80284-8

Hirasawa, T., and Shimizu, H. (2016). Recent advances in amino acid production by microbial cells. Curr. Opin. Biotechnol. 42, 133-146. doi: 10.1016/j.copbio. 2016.04.017

Hobbs, K. T. C., and Tartoff, K. (1987). Improved media for growing plasmid and cosmid clones. Focus 9, 9-12.

Hwang, C. S., Choi, E. S., Han, S. S., and Kim, G. J. (2012). Screening of a highly soluble and oxygen-independent blue fluorescent protein from metagenome. Biochem. Biophys. Res. Commun. 419, 676-681. doi: 10.1016/j.bbrc.2012.02.075

Kabus, A., Georgi, T., Wendisch, V. F., and Bott, M. (2007). Expression of the Escherichia coli pntAB genes encoding a membrane-bound transhydrogenase in Corynebacterium glutamicum improves L-lysine formation. Appl. Microbiol. Biotechnol. 75, 47-53. doi: 10.1007/s00253-006-0804-9

Kocharin, K., Siewers, V., and Nielsen, J. (2013). Improved polyhydroxybutyrate production by Saccharomyces cerevisiae through the use of the phosphoketolase pathway. Biotechnol. Bioeng. 110, 2216-2224. doi: 10.1002/bit.24888

Krömer, J. O., Bolten, C. J., Heinzle, E., Schröder, H., and Wittmann, C. (2008). Physiological response of Corynebacterium glutamicum to oxidative stress induced by deletion of the transcriptional repressor McbR. Microbiology 154, 3917-3930. doi: 10.1099/mic.0.2008/021204-0 
Laemmli, U. K. (1970). Cleavage of structural proteins during the assembly of the head of bacteriophage T4. Nature 227, 680-685. doi: 10.1038/227 $680 \mathrm{a} 0$

Lin, J.-L., Wagner, J. M., and Alper, H. S. (2017). Enabling tools for highthroughput detection of metabolites: metabolic engineering and directed evolution applications. Biotechnol. Adv. 35, 950-970. doi: 10.1016/j.biotechadv. 2017.07.005

Lindner, S. N., Petrov, D. P., Hagmann, C. T., Henrich, A., Krämer, R., Eikmanns, B. J., et al. (2013). Phosphotransferase system-mediated glucose uptake is repressed in phosphoglucoisomerase-deficient Corynebacterium glutamicum strains. Appl. Environ. Microbiol. 79, 2588-2595. doi: 10.1128/AEM.03231-12

Liu, D., Evans, T., and Zhang, F. (2015). Applications and advances of metabolite biosensors for metabolic engineering. Metab. Eng. 31, 35-43. doi: 10.1016/j. ymben.2015.06.008

Liu, J., Li, H., Zhao, G., Caiyin, Q., and Qiao, J. (2018). Redox cofactor engineering in industrial microorganisms: strategies, recent applications and future directions. J. Ind. Microbiol. Biotechnol. 45, 313-327. doi: 10.1007/ s10295-018-2031-7

Liu, Y., and Wang, M. (2017). Design, optimization and application of small molecule biosensor in metabolic engineering. Front. Microbiol. 8:2012. doi: 10.3389/fmicb.2017.02012

Lu, W., Wang, L., Chen, L., Hui, S., and Rabinowitz, J. D. (2018). Extraction and quantitation of nicotinamide adenine dinucleotide redox cofactors. Antioxid. Redox Signal. 28, 167-179. doi: 10.1089/ars.2017.7014

Mailloux, R. J., Lemire, J., and Appanna, V. D. (2011). Metabolic networks to combat oxidative stress in Pseudomonas fluorescens. Antonie Van Leeuwenhoek 99, 433-442. doi: 10.1007/s10482-010-9538-x

Marx, A., De Graaf, A. A., Wiechert, W., Eggeling, L., and Sahm, H. (1996). Determination of the fluxes in the central metabolism of Corynebacterium glutamicum by nuclear magnetic resonance spectroscopy combined with metabolite balancing. Biotechnol. Bioeng. 49, 111-129. doi: 10.1002/(SICI)10970290(19960120)49:2<111::AID-BIT1 > 3.0.CO;2-T

Marx, A., Hans, S., Mockel, B., Bathe, B., De Graaf, A. A., Mccormack, A. C., et al. (2003). Metabolic phenotype of phosphoglucose isomerase mutants of Corynebacterium glutamicum. J. Biotechnol. 104, 185-197. doi: 10.1016/S01681656(03)00153-6

Ng, C. Y., Farasat, I., Maranas, C. D., and Salis, H. M. (2015). Rational design of a synthetic entner-doudoroff pathway for improved and controllable NADPH regeneration. Metab. Eng. 29, 86-96. doi: 10.1016/j.ymben.2015. 03.001

Niesen, F. H., Berglund, H., and Vedadi, M. (2007). The use of differential scanning fluorimetry to detect ligand interactions that promote protein stability. Nat. Protoc. 2, 2212-2221.

Polizzi, K. M., Moore, D. A., and Bommarius, A. S. (2007). A short-chain dehydrogenase/reductase from Vibrio vulnificus with both blue fluorescence and oxidoreductase activity. Chem. Commun. 18, 1843-1845.

Sambrook, J., Russel, D. W., Irwin, N., and Janssen, U. A. (2001). Molecular Cloning: A Laboratory Manual. Cold Spring Harbor, NY: Cold Spring Harbor Laboratory Press.

Sanchez, A. M., Andrews, J., Hussein, I., Bennett, G. N., and San, K. Y. (2006). Effect of overexpression of a soluble pyridine nucleotide transhydrogenase (UdhA) on the production of poly(3-hydroxybutyrate) in Escherichia coli. Biotechnol. Prog. 22, 420-425. doi: 10.1021/bp050375u

Sauer, U., Canonaco, F., Heri, S., Perrenoud, A., and Fischer, E. (2004). The soluble and membrane-bound transhydrogenases UdhA and PntAB have divergent functions in NADPH metabolism of Escherichia coli. J. Biol. Chem. 279, 66136619. doi: $10.1074 /$ jbc.M311657200

Schallmey, M., Frunzke, J., Eggeling, L., and Marienhagen, J. (2014). Looking for the pick of the bunch: high-throughput screening of producing microorganisms with biosensors. Curr. Opin. Biotechnol. 26, 148-154. doi: 10.1016/j.copbio. 2014.01.005

Siedler, S., Schendzielorz, G., Binder, S., Eggeling, L., Bringer, S., and Bott, M. (2014). SoxR as a single-cell biosensor for NADPH-consuming enzymes in Escherichia coli. ACS Synth. Biol. 3, 41-47. doi: 10.1021/sb40 $0110 \mathrm{j}$
Spaans, S. K., Weusthuis, R. A., Van Der Oost, J., and Kengen, S. W. (2015). NADPH-generating systems in bacteria and archaea. Front. Microbiol. 6:742. doi: $10.3389 /$ fmicb. 2015.00742

Studier, F. W., and Moffatt, B. A. (1986). Use of bacteriophage T7 RNA polymerase to direct selective high-level expression of cloned genes. J. Mol. Biol. 189, 113-130. doi: 10.1016/0022-2836(86)90385-2

Tao, R., Zhao, Y., Chu, H., Wang, A., Zhu, J., Chen, X., et al. (2017). Genetically encoded fluorescent sensors reveal dynamic regulation of NADPH metabolism. Nat. Methods 14, 720-728. doi: 10.1038/nmeth.4306

Tauch, A., Kirchner, O., Loffler, B., Gotker, S., Pühler, A., and Kalinowski, J. (2002). Efficient electrotransformation of Corynebacterium diphtheriae with a minireplicon derived from the Corynebacterium glutamicum plasmid pGA1. Curr. Microbiol. 45, 362-367. doi: 10.1007/s00284-002-3728-3

Wang, C., Zhou, Z., Cai, H., Chen, Z., and Xu, H. (2017). Redirecting carbon flux through pgi-deficient and heterologous transhydrogenase toward efficient succinate production in Corynebacterium glutamicum. J. Ind. Microbiol. Biotechnol. 44, 1115-1126. doi: 10.1007/s10295-017-1933-0

Wang, Z., Chan, S. H. J., Sudarsan, S., Blank, L. M., Jensen, P. R., and Solem, C. (2016). Elucidation of the regulatory role of the fructose operon reveals a novel target for enhancing the NADPH supply in Corynebacterium glutamicum. Metab. Eng. 38, 344-357. doi: 10.1016/j.ymben.2016.08.004

Weckbecker, A., and Hummel, W. (2004). Improved synthesis of chiral alcohols with Escherichia coli cells co-expressing pyridine nucleotide transhydrogenase, NADP + -dependent alcohol dehydrogenase and NAD + -dependent formate dehydrogenase. Biotechnol. Lett. 26, 1739-1744. doi: 10.1007/s10529-004-37462

Wegner, A., Meiser, J., Weindl, D., and Hiller, K. (2015). How metabolites modulate metabolic flux. Curr. Opin. Biotechnol. 34, 16-22. doi: 10.1016/j.copbio.2014.11. 008

Wiederkehr, A., and Demaurex, N. (2017). Illuminating redox biology using NADH-and NADPH-specific sensors. Nat. Methods 14, 671-672. doi: 10.1038/ nmeth.4336

Xu, J.-Z., Yang, H.-K., and Zhang, W.-G. (2018). NADPH metabolism: a survey of its theoretical characteristics and manipulation strategies in amino acid biosynthesis. Crit. Rev. Biotechnol. 38, 1061-1076. doi: 10.1080/07388551.2018. 1437387

Ying, W. (2008). NAD + /NADH and NADP + /NADPH in cellular functions and cell death: regulation and biological consequences. Antioxid. Redox Signal. 10, 179-206. doi: 10.1089/ars.2007.1672

Zampieri, M., Sekar, K., Zamboni, N., and Sauer, U. (2017). Frontiers of highthroughput metabolomics. Curr. Opin. Chem. Biol. 36, 15-23. doi: 10.1016/j. cbpa.2016.12.006

Zhao, C., Zhao, Q., Li, Y., and Zhang, Y. (2017). Engineering redox homeostasis to develop efficient alcohol-producing microbial cell factories. Microb. Cell Fact. 16:115. doi: 10.1186/s12934-017-0728-3

Zhao, Y., Hu, Q., Cheng, F., Su, N., Wang, A., Zou, Y., et al. (2015). SoNar, a highly responsive NAD + /NADH sensor, allows high-throughput metabolic screening of anti-tumor agents. Cell Metab. 21, 777-789. doi: 10.1016/j.cmet. 2015.04.009

Zhao, Y., Wang, A., Zou, Y., Su, N., Loscalzo, J., and Yang, Y. (2016). In vivo monitoring of cellular energy metabolism using SoNar, a highly responsive sensor for NAD( + )/NADH redox state. Nat. Protoc. 11, 1345-1359. doi: 10.1038/nprot.2016.074

Conflict of Interest Statement: The authors declare that the research was conducted in the absence of any commercial or financial relationships that could be construed as a potential conflict of interest.

Copyright (c) 2018 Goldbeck, Eck and Seibold. This is an open-access article distributed under the terms of the Creative Commons Attribution License (CC BY). The use, distribution or reproduction in other forums is permitted, provided the original author(s) and the copyright owner(s) are credited and that the original publication in this journal is cited, in accordance with accepted academic practice. No use, distribution or reproduction is permitted which does not comply with these terms. 